\title{
Conhecimento dos \\ cirurgiões-dentistas em atendimento de pacientes com coagulopatias hereditárias
}

\author{
Knowledge of dentists in the care of patients with hereditary \\ coagulopathies
}

\author{
Rafaella Bastos Leite* \\ Rayssa Nunes da Mota Nascimento** \\ Renata de Souza Coelho Soares ${ }^{* *}$ \\ Andreza Cristina de Lima Targino Marssoni ${ }^{* * * *}$ \\ Carlos Augusto Galvão Barboza ${ }^{* * * *}$ \\ Raquel Cristina Barboza Gomes
}

\section{Resumo}

Objetivo: verificar o conhecimento dos cirurgiões-dentistas que trabalham nas Unidades Básicas de Saúde (UBS) com Equipes de Saúde Bucal (ESB) modalidade I, no município de Campina Grande, sobre o atendimento odontológico de pacientes com coagulopatias hereditárias. Sujeitos e método: por meio de questionário autoaplicável, foi feita uma entrevista com os cirurgiões-dentistas do município em questão para analisar os seus conhecimentos. Participaram da pesquisa 24 profissionais que se encaixaram nos critérios de inclusão e responderam de forma adequada o questionário com questões objetivas, sendo que os participantes foram orientados a assinalar somente uma alternativa para cada questionamento. Resultados: a maioria dos profissionais é formada há mais de 10 anos. A média de acertos das respostas foi de $50 \%$. Em relação aos achados clínicos que determinam a possível presença de distúrbio hemorrágico, 44\% afirmaram que são: púrpura, sangramento gengival espontâneo e hemartrose. Os pacientes considerados de risco elevado para o tratamento odontológico foram: pacientes sem distúrbios hemorrágicos revelados, mas com exames complementares alterados; pacientes em tratamento com AAS; e pacientes em tratamento com anticoagulante por via oral. A maioria (68\%) não considera a utilização de sugadores de saliva como risco para sangramento bucal. Os procedimentos odontológicos que os profissionais não se sentem seguros a executar, nesse tipo de paciente, foram: exodontias (88\%); tratamento periodontal ci- rúrgico (76\%); raspagem e alisamento coronoradicular (RACR) (28\%); anestesia do nervo alveolar inferior ou outros (24\%); tratamento endodôntico (20\%); e anestesia infiltrativa (8\%). Conclusão: os dados obtidos na pesquisa mostraram que o conhecimento dos cirurgiões-dentistas das UBS do município de Campina Grande não é satisfatório, havendo dúvidas sobre a maioria dos tratamentos odontológicos direcionados aos pacientes com coagulopatias hereditárias.

Palavras-chave: Coagulação sanguínea. Transtornos hemorrágicos. Transtornos hemostáticos.

\section{Introdução}

É possível conceituar paciente especial como todo indivíduo que apresenta alteração física, orgânica, intelectual, social ou emocional, podendo ser aguda ou crônica, simples ou complexa, que necessita de educação especial e instruções suplementares, temporária ou definitivamente ${ }^{1}$.

A coagulopatia é definida como uma "doença hemorrágica", resultante da deficiência quantitativa e/ou qualitativa de uma ou mais proteínas plasmáticas (fatores) da coagulação. Quando a coagulopatia é diagnosticada precocemente e tratada de modo adequado, a expectativa de vida do indivíduo que

\footnotetext{
Professora do Departamento de Odontologia da Universidade Estadual da Paraíba, Campina Grande, Paraíba, Brasil.

Acadêmica do Departamento de Odontologia da Universidade Estadual da Paraíba, Campina Grande, Paraíba, Brasil.

Professora do Departamento de Odontologia da Universidade Estadual da Paraíba, Campina Grande, Paraíba, Brasil.

** Professora do Departamento de Odontologia da Universidade Estadual da Paraíba, Campina Grande, Paraíba, Brasil.

***** Professor doutor do Programa de Pós-Graduação em Patologia Oral da Universidade Federal do Rio Grande do Norte, Natal, Rio Grande do Norte, Brasil.

Professora do Departamento de Odontologia da Universidade Estadual da Paraíba, Campina Grande, Paraíba, Brasil.
} 
nasce com essa patologia é similar à média da população ${ }^{2}$. No Brasil, a participação de cirurgiões-dentistas nas equipes multidisciplinares de atendimento aos pacientes portadores de coagulopatias tem possibilitado que o tratamento odontológico desses sujeitos seja ambulatorial, diminuindo consideravelmente as necessidades de reposição de fatores de coagulação ${ }^{1}$.

As coagulopatias hereditárias têm como característica comum a redução da formação de trombina, fator essencial para a coagulação do sangue ${ }^{2}$. Pacientes acometidos podem apresentar sangramentos de gravidade variável, espontâneos ou pós-traumáticos, presentes ao nascimento ou diagnosticados ocasionalmente ${ }^{3}$. No entanto, as coagulopatias hereditárias apresentam herança genética, quadros clínico e laboratorial distintos entre $\mathrm{si}^{2}$. A hemofilia e a doença de Von Willebrand são as mais comuns das coagulopatias hereditárias e devem ser detectadas antes que qualquer tratamento odontológico seja realizado ${ }^{3}$.

O profissional deve estar atento para um correto protocolo de atendimento, podendo utilizar, nesses casos, vários recursos hemostáticos, tais como o uso de selantes de fibrina, cimento cirúrgico, gelo, anestésico adequado, além do uso de ácido tricloroacético (ATA) $10 \%$ e antifibrinolíticos, evitando ao máximo complicações durante o procedimento a ser realizado ${ }^{4}$. O cirurgião-dentista deve estar ciente dos protocolos para cada tipo de tratamento, formulando um planejamento adequado para o tratamento periodontal, endodôntico, restaurador, protético, ortodôntico e cirúrgico ${ }^{4}$. O manejo de portadores de coagulopatias requer um diagnóstico preciso da deficiência de coagulação do paciente, bem como um planejamento juntamente com o hematologista para o pré, o trans e o pós-cirúrgico, além de um conhecimento adequado por parte do cirurgião-dentista sobre coagulopatia e seus métodos de controle ${ }^{3,4}$.

Portanto, o objetivo desta pesquisa foi verificar o conhecimento dos cirurgiões-dentistas que trabalham nas Unidades Básicas de Saúde (UBS) com Equipe de Saúde Bucal (ESB) modalidade I, no município de Campina Grande, Paraíba, sobre o atendimento odontológico de pacientes com coagulopatias hereditárias.

\section{Sujeitos e método}

Este estudo é descritivo e do tipo transversal, com análise de dados primários, envolvendo 33 cirurgiões-dentistas em atividade nas 48 UBS com ESB modalidade 1 do município de Campina Grande, Paraíba (população: 386.000 habitantes).

$\mathrm{O}$ universo da amostra foi de 33 profissionais, a amostra do tipo não probabilística foi obtida por conveniência, ou seja, integraram a pesquisa todos os cirurgiões-dentistas que trabalham em UBS com ESB modalidade I e que, por livre e espontânea vontade, aceitaram participar e assinar o termo de consentimento livre e esclarecido. A amostra final somou 24 participantes.

O presente estudo recebeu a aprovação do Comitê de Ética em Pesquisa com Seres Humanos da Universidade Estadual da Paraíba (Brasil), sob processo número 35555314.1.0000.5187, em complacência com a Resolução 466/12, do Conselho Nacional de Saúde e Ministério da Saúde e a Resolução UEPB/Consepe 10/2001. Recebeu também a autorização institucional para realização da pesquisa. Os direitos de todos os participantes foram protegidos.

O questionário autoaplicável continha doze questões objetivas, sendo duas pessoais, referente ao tempo de formação e ao procedimento que o profissional não se sente seguro a realizar, e dez questões referentes a: definição das diáteses hemorrágicas, sinais clínicos de distúrbios hemorrágicos, métodos de hemostasia da cavidade bucal, técnicas anestésicas, tratamento periodontal, tratamento endodôntico, sangramentos gengivais pós-esfoliação de dente decíduo e exodontias.

Como critérios de exclusão, foram eliminados os questionários que foram respondidos de forma inadequada, tendo assinalada mais de uma alternativa ou com questões não respondidas. As perguntas foram elaboradas de acordo com as orientações contidas no Manual de atendimento odontológico a pacientes com coagulopatias hereditárias, de 2008, do Ministério da Saúde. A aplicação do questionário durou aproximadamente 10 minutos.

Os dados foram analisados descritivamente por meio de distribuições absolutas. Foi utilizado o teste Qui-Quadrado de Pearson ou o teste Exato de Fisher, quando a condição para utilização do primeiro não foi observada para a verificação da hipótese de associação significativa entre as variáveis categóricas.

A margem de erro utilizada nas decisões dos testes estatísticos foi de 5\%. O programa estatístico utilizado para registro dos dados e obtenção dos cálculos estatísticos foi o Statistical Package for the Social Sciences (SPSS) versão 21.

\section{Resultados}

Um total de 24 cirurgiões-dentistas participou deste estudo, o que corresponde a uma taxa de resposta de $72,7 \%$. A exclusão de 12 profissionais foi devido a: recusa de participação na pesquisa $(n=8)$; férias $(n=2)$; licença maternidade $(n=1)$; questionário respondido de forma incorreta $(n=1)$. Mediante as respostas do questionário aplicado, foi feita a análise dos dados obtidos. Vale ressaltar que algumas das questões não foram respondidas por todos os participantes, como também, mesmo com orientação, houve marcação de mais de uma alternativa em questões que havia apenas uma alternativa correta; portanto, o número da amostra pode variar. 
Dos profissionais participantes, $20(83,3 \%)$ tinham mais de 10 anos de formação acadêmica. A média de acertos foi de 5 das 10 questões sobre o assunto, o que corresponde a 50\%. A Tabela 1 mostra a distribuição relativa das respostas dos cirurgiões- -dentistas para cada uma das questões em que havia apenas uma opção correta. A Tabela 2 mostra a avaliação das questões relacionadas aos pacientes com coagulopatias hereditárias, segundo o tempo de formado (até 15 anos e mais de 15 anos).

Tabela 1 - Avaliação das respostas dos cirurgiões-dentistas para questões com apenas uma resposta correta, distribuída em frequência relativa

\begin{tabular}{l|c|c|c}
\hline \multicolumn{1}{c|}{ Questão } & N* & Correto & Incorreto \\
\hline Em relação às diáteses hemorrágicas & 18 & 08 & 10 \\
\hline Achados clínicos que determinam a possível presença de distúrbio hemorrágico & 23 & 11 & 12 \\
\hline São considerados pacientes de risco elevado para o tratamento odontológico & 24 & 13 & 11 \\
\hline São métodos auxiliares na hemostasia da cavidade bucal, exceto & 24 & 16 & 08 \\
\hline De acordo com as técnicas anestésicas & 23 & 05 & 18 \\
\hline Quanto ao tratamento periodontal em pacientes com coagulopatias hereditárias & 18 & 18 & - \\
\hline São procedimentos que aumentam o risco de sangramento, exceto & 24 & 05 & 19 \\
\hline Assinale a alternativa correta, quanto ao tratamento endodôntico & 22 & 13 & 09 \\
\hline Diante da necessidade de extração dentária & 22 & 07 & 15 \\
\hline
\end{tabular}

*Número de cirurgiões-dentistas que responderam as questões.

Fonte: autores.

Tabela 2 - Avaliação das questões relacionadas aos pacientes com coagulopatias hereditárias segundo o tempo de formado

\begin{tabular}{|c|c|c|c|c|c|c|c|}
\hline \multicolumn{8}{|c|}{ Tempo de formado (anos) } \\
\hline \multirow{2}{*}{ Variável } & \multicolumn{2}{|c|}{ Até 15} & \multicolumn{2}{|c|}{ Mais de 15} & \multicolumn{2}{|c|}{ Grupo Total } & \multirow{3}{*}{ Valor de $p$} \\
\hline & $\mathrm{n}$ & $\%$ & $\mathrm{n}$ & $\%$ & $\mathrm{n}$ & $\%$ & \\
\hline Total & 12 & 100 & 12 & 100 & 24 & 100 & \\
\hline $\begin{array}{l}\text { - Em relação às diáteses hemorrágicas, assinale a alternativa correta: } \\
\text { Resposta certa } \\
\text { Resposta errada }\end{array}$ & $\begin{array}{l}5 \\
7 \\
\end{array}$ & $\begin{array}{l}41,7 \\
58,3\end{array}$ & $\begin{array}{l}3 \\
9 \\
\end{array}$ & $\begin{array}{l}25,0 \\
75,0\end{array}$ & $\begin{array}{c}8 \\
16 \\
\end{array}$ & $\begin{array}{l}33,3 \\
66,7\end{array}$ & $\mathrm{p}^{(1)}=0,667$ \\
\hline $\begin{array}{l}\text { - Assinale a alternativa correta que contém os achados clínicos que } \\
\text { determinam a possível presença de distúrbios hemorrágicos? } \\
\text { Resposta certa } \\
\text { Resposta errada }\end{array}$ & $\begin{array}{l}6 \\
6\end{array}$ & $\begin{array}{l}50,0 \\
50,0\end{array}$ & $\begin{array}{l}5 \\
7\end{array}$ & $\begin{array}{l}41,7 \\
58,3\end{array}$ & $\begin{array}{l}11 \\
13\end{array}$ & $\begin{array}{l}45,8 \\
54,2\end{array}$ & $\mathrm{p}^{(2)}=0,682$ \\
\hline $\begin{array}{l}\text { São considerados pacientes de risco elevado para o tratamento } \\
\text { odontológico: } \\
\text { Resposta certa } \\
\text { Resposta errada } \\
\end{array}$ & $\begin{array}{l}6 \\
6\end{array}$ & $\begin{array}{l}50,0 \\
50,0\end{array}$ & $\begin{array}{l}7 \\
5\end{array}$ & $\begin{array}{l}58,3 \\
41,7\end{array}$ & $\begin{array}{l}13 \\
11\end{array}$ & $\begin{array}{l}54,2 \\
45,8\end{array}$ & $\mathrm{p}^{(2)}=0,682$ \\
\hline $\begin{array}{l}\text { - São métodos auxiliares à hemostasia da cavidade bucal: } \\
\text { Resposta certa } \\
\text { Resposta errada }\end{array}$ & $\begin{array}{l}9 \\
3 \\
\end{array}$ & $\begin{array}{l}75,0 \\
25,0 \\
\end{array}$ & $\begin{array}{l}7 \\
5 \\
\end{array}$ & $\begin{array}{l}58,3 \\
41,7 \\
\end{array}$ & $\begin{array}{c}16 \\
8 \\
\end{array}$ & $\begin{array}{l}66,7 \\
33,3 \\
\end{array}$ & $\mathrm{p}^{(1)}=0,667$ \\
\hline $\begin{array}{l}\text { De acordo com as técnicas anestésicas, sabe-se que: } \\
\text { Resposta certa } \\
\text { Resposta errada }\end{array}$ & $\begin{array}{c}2 \\
10\end{array}$ & $\begin{array}{l}16,7 \\
83,3\end{array}$ & $\begin{array}{l}3 \\
9\end{array}$ & $\begin{array}{l}25,0 \\
75,0\end{array}$ & $\begin{array}{c}5 \\
19\end{array}$ & $\begin{array}{l}20,8 \\
79,2\end{array}$ & $\mathrm{p}^{(1)}=1,000$ \\
\hline $\begin{array}{l}\text { Quanto ao tratamento periodontal em pacientes com coagulopatia } \\
\text { hereditária, é correto afirmar: } \\
\text { Resposta certa } \\
\text { Resposta errada }\end{array}$ & $\begin{array}{l}9 \\
3\end{array}$ & $\begin{array}{l}75,0 \\
25,0\end{array}$ & $\begin{array}{l}9 \\
3\end{array}$ & $\begin{array}{l}75,0 \\
25,0\end{array}$ & $\begin{array}{c}18 \\
6\end{array}$ & $\begin{array}{l}75,0 \\
25,0\end{array}$ & $\mathrm{p}^{(1)}=1,000$ \\
\hline $\begin{array}{l}\text { - São procedimentos que aumentam o risco de sangramento bucal, exceto: } \\
\text { Resposta certa } \\
\text { Resposta errada }\end{array}$ & $\begin{array}{c}2 \\
10\end{array}$ & $\begin{array}{l}16,7 \\
83,3\end{array}$ & $\begin{array}{l}3 \\
9\end{array}$ & $\begin{array}{l}25,0 \\
75,0\end{array}$ & $\begin{array}{c}5 \\
19\end{array}$ & $\begin{array}{l}20,8 \\
79,2\end{array}$ & $\mathrm{p}^{(1)}=1,000$ \\
\hline $\begin{array}{l}\text { Assinale a alternativa correta: } \\
\text { Resposta certa } \\
\text { Resposta errada } \\
\end{array}$ & $\begin{array}{l}8 \\
4 \\
\end{array}$ & $\begin{array}{l}66,7 \\
33,3 \\
\end{array}$ & $\begin{array}{l}5 \\
7 \\
\end{array}$ & $\begin{array}{l}41,7 \\
58,3 \\
\end{array}$ & $\begin{array}{l}13 \\
11 \\
\end{array}$ & $\begin{array}{l}54,2 \\
45,8 \\
\end{array}$ & $\mathrm{p}^{(2)}=0,219$ \\
\hline $\begin{array}{l}\text { Nos casos de sangramentos gengivais pós-esfoliação de dente decíduo, } \\
\text { deve ser feito: } \\
\text { Resposta errada } \\
\text { Resposta incompleta }\end{array}$ & $\begin{array}{l}3 \\
9\end{array}$ & $\begin{array}{l}25,0 \\
75,0\end{array}$ & $\begin{array}{l}6 \\
6\end{array}$ & $\begin{array}{l}50,0 \\
50,0\end{array}$ & $\begin{array}{c}9 \\
15\end{array}$ & $\begin{array}{l}37,5 \\
62,5\end{array}$ & $\mathrm{p}^{(1)}=0,400$ \\
\hline $\begin{array}{l}\text { Diante da necessidade de extração dentária, deve ser feito: } \\
\text { Resposta certa } \\
\text { Resposta errada }\end{array}$ & $\begin{array}{l}4 \\
8\end{array}$ & $\begin{array}{l}33,3 \\
66,7\end{array}$ & $\begin{array}{l}3 \\
9\end{array}$ & $\begin{array}{l}25,0 \\
75,0\end{array}$ & $\begin{array}{c}7 \\
17\end{array}$ & $\begin{array}{l}29,2 \\
70,8\end{array}$ & $\mathrm{p}^{(1)}=1,000$ \\
\hline $\begin{array}{l}\text { Quais dos seguintes procedimentos não se sente seguro em realizar em } \\
\text { pacientes com coagulopatia hereditária: } \\
\text { Anestesia infiltrativa } \\
\text { Anestesia do nervo alveolar ou outros } \\
\text { Tratamento periodontal (RACR) } \\
\text { Tratamento periodontal cirúrgico } \\
\text { Tratamento endodôntico } \\
\text { Exodontia } \\
\end{array}$ & $\begin{array}{c}2 \\
3 \\
5 \\
8 \\
4 \\
10\end{array}$ & $\begin{array}{l}16,7 \\
25,0 \\
41,7 \\
66,7 \\
33,3 \\
83,3\end{array}$ & $\begin{array}{c}- \\
3 \\
4 \\
10 \\
1 \\
10\end{array}$ & $\begin{array}{c}- \\
25,0 \\
33,3 \\
83,3 \\
8,3 \\
83,3\end{array}$ & $\begin{array}{c}2 \\
6 \\
9 \\
18 \\
5 \\
20\end{array}$ & $\begin{array}{c}8,3 \\
25,0 \\
37,5 \\
75,0 \\
20,8 \\
83,3\end{array}$ & $\begin{array}{l}\mathrm{p}^{(1)}=0,093 \\
\mathrm{p}^{(1)}=0,556 \\
\mathrm{p}^{(1)}=0,360 \\
\mathrm{p}^{(1)}=0,525 \\
\mathrm{p}^{(1)}=0,083 \\
\mathrm{p}^{(1)}=0,230\end{array}$ \\
\hline
\end{tabular}

(1): teste Exato de Fisher.

(2): teste Qui-Quadrado de Pearson.

Fonte: autores. 
Em relação às diáteses hemorrágicas, 8 (33,3\%) profissionais afirmaram que a hemofilia é uma condição hematológica caracterizada pela deficiência de fator VIII, enquanto 8 (33,3\%) consideraram que trombocitopatia é uma condição patológica hereditária relacionada com a deficiência de fatores de coagulação e 6 (25\%) não responderam a questão.

Quando questionados sobre os achados clínicos que determinam a possível presença de distúrbio hemorrágico: 11 (45,8\%) afirmaram que são púrpura, sangramento gengival espontâneo e hemartrose; $5(20,8 \%)$ consideraram como sendo hematoma, sangramento gengival e petéquias. Quanto aos pacientes de risco elevado para o tratamento odontológico, as alternativas mais assinaladas foram: pacientes em tratamento com ácido acetilsalicílico (36\%); pacientes em tratamento com anticoagulantes por via oral (28\%); e pacientes sem distúrbios hemorrágicos revelados, mas com os exames complementares anormais $(54,2 \%)$.

Em relação aos métodos auxiliares na hemostasia da cavidade bucal, $16(66,7 \%)$ afirmaram que $o$ ácido tricloroacético (ATA 15\%) não é utilizado. Outro ponto questionado foi sobre técnicas anestésicas, em que $16(66,7 \%)$ consideraram que, após uma anestesia infiltrativa que resultou na formação de hematomas, deve-se indicar crioterapia, durante as primeiras 48 horas; 3 (12\%) responderam que a prescrição de fatores de coagulação deficiente deve ser feita exclusivamente pelo cirurgião-dentista responsável; $5(20,8 \%)$ responderam que se deve elevar previamente o fator de coagulação deficiente a $30 \%$, na necessidade de técnica anestésica de bloqueio do nervo alveolar inferior; e $5(20 \%)$ que se deve elevar previamente o fator de coagulação deficiente a $50 \%$, na necessidade de técnica anestésica de bloqueio do nervo alveolar inferior.

No que se refere ao tratamento periodontal, 18 (75\%) afirmaram que os procedimentos cirúrgicos periodontais apresentam necessidade do preparo prévio do paciente, com indicação da reposição dos fatores de coagulação e da associação do antifibrinolíticos, e $4(16 \%)$ não responderam a questão. Quanto aos procedimentos que não aumentam o risco de sangramento bucal, as alternativas mais assinaladas foram: utilização de sugadores de saliva $(66,7 \%)$; utilização de fios retratores embebidos $(20,8 \%)$; e utilização de diques de borracha e matrizes $(12,5 \%)$.

Quando questionados sobre o tratamento endodôntico, 13 (54,2\%) afirmaram que, nos casos em que houver sangramento persistente nas biopulpectomias, pode ser feita compressão intracanal com pasta de hidróxido de cálcio; $5(20,8 \%)$ responderam que, no tratamento endodôntico, nunca há necessidade da reposição dos fatores de coagulação. Tratando-se do que se deve fazer em caso de sangramento gengival pós-esfoliação de dente decíduo, 4 $(16,6 \%)$ afirmaram que devem ser feitas curetagem do tecido de granulação remanescente e sutura, se possível; 5 (20,8\%) marcaram apenas sutura; 6 (25\%) escolheram administração de agente hemostático via oral e odontológico; 7 (29,2\%) optaram por administração de agentes hemostáticos via oral e proteção da região com cimento cirúrgico; e $2(8,3 \%)$ não responderam.

Diante da necessidade de extração dentária, 15 $(62,5 \%)$ afirmaram que se deve elevar o fator de coagulação deficiente a $50 \%$ e associar terapia de reposição dos fatores de coagulação a outros métodos de hemostasia local; $7(29,2 \%)$ que se deve elevar o fator de coagulação deficiente a $80 \%$ e associar terapia de reposição dos fatores de coagulação a outros métodos de hemostasia local; e $2(8,3 \%)$ não responderam a questão.

Os procedimentos dentários que os participantes afirmaram não ter segurança em executar foram: anestesia infiltrativa $(8,3 \%)$; anestesia do nervo alveolar inferior e outros (25\%); tratamento periodontal - RACR $(37,5 \%)$; tratamento periodontal cirúrgico $(75 \%)$; tratamento endodôntico (20,8\%); e exodontias $(83,3 \%)$. A opção de tratamento restaurador não foi assinalada por nenhum dos profissionais.

\section{Discussão}

Os distúrbios de sangramento são condições que alteram a capacidade de vasos sanguíneos, plaquetas e fatores de coagulação de promover a hemostasia. Os distúrbios hereditários de sangramento são geneticamente transmitidos ${ }^{5}$, sendo a doença de Von Willebrand (DvW) a mais comum, com prevalência de $1 \%$ da população; de acordo com estatísticas internacionais, essa doença é usualmente um traço de herança autossômica dominante ${ }^{6}$. No Brasil, não se conhece a prevalência dessa doença, embora ela pareça ser subdiagnosticada, uma vez que o número de casos de DvW registrado é bastante inferior ao de pacientes com hemofilia ${ }^{7}$.

A hemofilia A, deficiência do fator VIII, ocorre em 1 a cada 5.000 indivíduos do sexo masculino, e a hemofilia B (doença de Christmas), deficiência de fator IX, é encontrada em 1 a cada $30.000^{3,7}$. Cerca de $80 \%$ de todos os distúrbios são hemofilia $\mathrm{A}, 13 \%$ hemofilia B e 6\% são deficiências do fator XI (muito rara $)^{8}$. No Brasil, existem cerca de 10.000 pacientes com hemofilia A e $\mathrm{B}^{9}$.

De acordo com os achados clínicos que determinam a possível presença de distúrbio hemorrágico, 33,3\% dos pesquisados afirmaram, concordando com a literatura, que pacientes acometidos por coagulopatias hereditárias podem apresentar sangramentos de gravidade variável, espontâneos ou pós-traumáticos, presentes ao nascimento ou diagnosticados ocasionalmente (em pré-operatório ou exame periódico). Aqueles acometidos por hemofilia podem ter sangramentos de gravidade variável dependendo do nível de atividade do fator presente no plasma. As hemorragias podem ser espontâne- 
as ou pós-traumáticas, presentes ao nascimento ou somente diagnosticadas ocasionalmente. Na hemofilia grave, os sangramentos aparecem, em geral, antes do segundo ano de vida. As manifestações clínicas mais características da doença são hemartroses e hematomas. Os pacientes com DvW de leve intensidade são, em geral, assintomáticos, sendo, não raro, diagnosticados após trauma ou cirurgia. Indivíduos sintomáticos apresentam frequentemente sangramentos cutaneomucosos, tais como equimoses, epistaxe, menorragia, hemorragias gengival e digestiva ${ }^{3,5,10}$.

Vários métodos auxiliares na hemostasia da cavidade bucal podem ser utilizados ${ }^{9}$. Dos profissionais participantes da pesquisa, 66,7\% afirmaram que ácido tricloroacético (ATA 15\%) não é utilizado como método auxiliar, estando em concordância com a literatura, pois o agente cauterizante utilizado é o ácido tricloroacético (ATA 10\%). Em relação ao tratamento periodontal, os polimentos coronários devem ser realizados de maneira rotineira ${ }^{10}$. Sangramentos podem ser controlados localmente com compressão de gaze embebida em antifibrinolíticos, bolinhas de algodão embebidas em ATA a $10 \%$ ou cimento cirúrgico ${ }^{10}$.

Embora $1(4,17 \%)$ dos questionados tenha contraindicado esse procedimento, há autores que afirmam que a curetagem das bolsas periodontais deve ser feita de maneira gradativa, sem necessidade da terapia prévia de reposição dos fatores de coagulação $0^{7,8}$. Os antifibrinolíticos devem ser utilizados nos tratamentos periodontais, tanto na forma sistêmica como na local ${ }^{7}$. O selante de fibrina (SF) pode ser empregado nos casos em que se apresentem bolsas periodontais profundas ${ }^{10}$. $\mathrm{O}$ uso coadjuvante de substâncias antissépticas durante o tratamento periodontal deve ser indicado quando necessário ${ }^{5}$. Concordando com a literatura, 75\% dos participantes desta pesquisa afirmaram que os procedimentos cirúrgicos periodontais devem ser considerados como procedimentos de elevado risco de sangramento. Portanto, há necessidade do preparo prévio do paciente, com indicação da reposição dos fatores de coagulação e da associação dos antifibrinolíticos. Nesses casos, a utilização de meios hemostáticos locais (tais como o SF, o cimento cirúrgico e outros) deve ser indicada ${ }^{3,11}$.

Com relação à técnica do bloqueio do tronco alveolar inferior, recomenda-se que a utilização seja precedida por reposição dos fatores de coagulação, a fim de se elevar o fator deficiente a 30\%, $5(20,8 \%)$ profissionais afirmaram isso nesta pesquisa. Essa recomendação é feita devido à possibilidade de sangramento na região retromolar, com presença de trismo e risco de asfixia. A formação de hematomas decorrentes das técnicas anestésicas infiltrativas é rara. Nesse caso, deve-se usar gelo (macerado) em recipiente apropriado, que deve ser mantido no local por 20 minutos, com intervalos de 20 minutos de repouso, durante as primeiras 24 horas $^{3,12}$. Nesta pesquisa, $66,7 \%$ recomendaram crioterapia durante as primeiras 48 horas, mas não há justificativa quanto à necessidade.
Alguns cuidados devem ser seguidos durante o tratamento odontológico dos pacientes com coagulopatias, como o uso de sugadores e de bombas a vácuo: cuidado com as mucosas livres e com o risco de formação de hematomas; pequenos sangramentos provenientes da colocação de matrizes ou cunhas de madeira podem ser controlados com compressão local, água fria e ATA a $20 \%{ }^{3}$. Neste estudo, a maioria $(66,7 \%)$ dos cirurgiões-dentistas não considerou o uso de sugadores de saliva como risco na formação de sangramento gengival. Fio retrátil embebido em solução hemostática pode ser usado na retração da mucosa quando houver cáries subgengivais ou mesmo para conter pequenos sangramentos.

A prescrição dos fatores de coagulação deve ser feita exclusivamente pelo médico hematologista e, sempre que possível, após discussão do caso com o cirurgião-dentista responsável ${ }^{3}$. É importante orientar o paciente e, quando for criança, o seu responsável. Na presença de qualquer intercorrência, o médico responsável deve ser comunicado para avaliar a necessidade da reposição do fator de coagulação. Diante da necessidade de exodontias, deve-se elevar o fator de coagulação deficiente a $80 \%$ e associar terapia de reposição dos fatores de coagulação a outros métodos da hemostasia local ${ }^{3}$. Apenas 7 $(29,2 \%)$ participantes desta pesquisa concordaram com a literatura, enquanto $15(62,5 \%)$ consideraram que o fator deficiente deve ser elevado a apenas $50 \%$, em associação com outros métodos de hemostasia local.

Um grande número de procedimentos realizados na odontologia pode causar sangramentos. Em circunstâncias normais, esses procedimentos podem ser realizados com um risco mínimo para o paciente $^{5}$. Quando questionados sobre os procedimentos que não se sentiram seguros para realizar, os participantes assinalaram as exodontias e o tratamento periodontal cirúrgico como os mais temidos $83,3 \%$ e $75 \%$, respectivamente, devido ao grande risco de sangramento; seguidos de tratamento periodontal RACR $(37,5 \%)$, anestesia do nervo alveolar inferior e outros $(25 \%)$, tratamento endodôntico $(20,5 \%)$ e anestesia infiltrativa (8,3\%). A opção de tratamento restaurador não foi assinalada por nenhum profissional, provavelmente por não mostrar risco de sangramento como as opções assinaladas.

Dos cirurgiões-dentistas participantes desta pesquisa, $20(83,3 \%)$ tinham mais de 10 anos de formação acadêmica. A média de acertos no questionário aplicado foi de 5 das 10 questões sobre o assunto, o que corresponde a 50\%. Esse índice revela o pouco conhecimento dos profissionais, o que poderia ser explicado por uma formação falha desses cirurgiões-dentistas. A Tabela 2 mostra a avaliação das questões relacionadas aos pacientes com coagulopatias hereditárias, segundo o tempo de formado (até 15 anos e mais de 15 anos). Os dados mostraram que não houve diferença significativa nos acertos das questões em relação ao tempo de formado, havendo, praticamente, um empate. 
Os resultados desta pesquisa enfatizaram a necessidade da capacitação dos profissionais envolvidos para um melhor controle da saúde bucal associada a problemas sistêmicos, a fim de elevar a qualidade de vida dos pacientes, evitar o foco apenas nos problemas de caráter oral e desenvolver uma atenção integral de qualidade. Para tanto, o diagnóstico é de extrema relevância, já que a boca é uma região altamente vascularizada, permitindo ao profissional de odontologia ser um dos primeiros a verificar distúrbios da homeostasia.

\section{Conclusão}

Os dados obtidos nesta pesquisa evidenciam que o conhecimento dos cirurgiões-dentistas das UBS do município de Campina Grande é pouco satisfatório e que eles têm dúvidas sobre a maioria dos tratamentos odontológicos direcionados aos pacientes com coagulopatias hereditárias. Diante disso, é necessária uma atualização para a capacitação de tais profissionais.

\section{Abstract}

Objective: this study aimed to determine the knowledge of dentists working in UBS with ESB mode I in the city of Campina Grande on Patients with hereditary Coagulopathies. Through self-administered questionnaire, own and without exclusion criteria, an interview was conducted with dental surgeons of the municipality concerned to analyze their knowledge of the relevant topic. By itself and without exclusion criterion an interview was made with the dentists concerning to analyze their knowledge about hereditary coagulopathies. Subjects and method: the participants were 24 dentists which fit the inclusion criteria and responded adequately to the questionnaire which contained objective questions, and the respondents were asked to point out only one alternative of each questioning. Results: most of them were graduated over 10 years. The mean score was $50 \%$ of the questionnaire. In relation to clinical findings that determine the possible presence of bleeding disorder, $44 \%$ said they are: purple spontaneous gingival bleeding and hemarthrosis; patients considered at high risk for dental treatment were patients without bleeding disorders disclosed but with altered exams; patients being treated with ASA; and patients treated with anticoagulant orally. Most dentists (68\%) do not consider the use of saliva-sucking as a risk for oral bleeding. Dental procedures that professionals do not feel safe running in those patients were: extractions $(88 \%)$; surgical periodontal treatment $(76 \%)$; RACR $(28 \%)$; anesthesia of nerve alveolar inferior or other $(24 \%)$; endodontic treatment $(20 \%)$; and infiltrative anesthesia $(8 \%)$. Conclusion: the data obtained from the survey showed that knowledge of dentists from Campina Grande municipality is not satisfactory and there is doubt on most dental treatments targeted to patients with inherited bleeding disorders.

Keywords: Blood coagulation. Hemorrhagic disorders. Hemostatic disorders.

\section{Referências}

1. Peres AS, Peres SHCS, Silva RHA. Atendimento a pacientes especiais: reflexão sobre os aspectos éticos e legais. Rev Fac Odontol Lins 2005; 17(1):49-53.

2. Brasil. Ministério da Saúde. Secretaria de Atenção à Saúde. Departamento de Atenção Especializada. Manual de tratamento das coagulopatias hereditárias. Brasília: Ministério da Saúde; 2006.

3. Brasil. Ministério da Saúde. Secretaria de Atenção à Saúde. Departamento de Atenção Especializada. Manual de atendimento odontológico a pacientes com coagulopatias hereditárias. Brasília: Ministério da Saúde; 2008.

4. Dall M, Ribeiro AA, Shenkel A, Samuelsson M, Studzinski MS, Almeida D. Manejo odontológico de pacientes com coagulopatias - revisão de literatura e relato de caso: síndrome de Bernard Soulier. RFO UPF 2011; 16(2):193-9.

5. Little JW, Falace DA, Rhouds NL, Miller CS. Manejo odontológico do paciente clinicamente comprometido. Rio de Janeiro: Elsevier; 2008.

6. Kessler CM. Hemorrhagic disorders: coagulation fator deficiences. In: Little JW, Falace DA, Miller CS, Rhodus NL. Manejo odontológico do paciente clinicamente comprometido. 7 . ed. Rio de Janeiro: Elsevier; 2008. p. 344-348.

7. Castaman G, Federici AB, Rodeghiero F, Mannucci PM. Von Willebrand's disease in the year 2003: towards the complete identification of gene defects for correct diagnosis and treatment. Haematologica 2003; 88:94-108.

8. Rodgers G, Greenberg CS. Inherited coagulation disorders. In: Little JW, Falace DA, Miller CS, Rhodus NL. Manejo odontológico do paciente clinicamente comprometido. 7. ed. Rio de Janeiro: Elsevier; 2008. p. 381-414.

9. Rezende SM. Distúrbios da hemostasia: doenças hemorrágicas. Rev Med Minas Gerais 2010; 20(4):534-53.

10. Federici AB, Castaman G, Mannucci PM. For the Italian Association of Hemophilia Centers. Guidelines for the diagnosis and management of von Willebrand disease. In: Rezende SM. Distúrbios da hemostasia: doenças hemorrágicas. Rev Med 2010; 20(4):534-53.

11. Silva SCS, Silva ESC, Menezes KT, Lira JR, Brito RLB. Tratamento periodontal de paciente hemofílico: relato de caso. Rev Bras Cienc Saúde 2012; 16(2):243-8.

12. Hartman MJ, Caccamese JF, Bergman SA. Perioperative management of a patient with Bernard Soulier syndrome for third molar surgery. Oral Surg Oral Med Oral Pathol Oral Radiol Endod 2007; 103(5):626-9.

\section{Endereço para correspondência:}

Rafaella Bastos Leite

Av. Salgado Filho, 1787, Lagoa Nova

59056-000, Natal, RN, Brasil

Telefones: (84) 32154138 - (83) 998092884

E-mail: rrafaella_bastos@hotmail.com

Recebido: 30/05/18. Aceito: 19/07/18. 